\title{
Cylinder Pressure and Combustion Heat Release Estimation for SI Engine Diagnostics Using Nonlinear Sliding Observers
}

\author{
Yaojung Shiao and John J. Moskwa
}

\begin{abstract}
Cylinder pressure is an important parameter in engine combustion analysis or engine diagnosis. An approach is introduced to estimate cylinder pressure and combustion heat release in multicylinder SI engines based solely on engine speed measurements. Because of the nonlinear nature of engines, this estimation employs a nonlinear observer: the Sliding Observer.

In many applications, cylinder pressure is critical for control or engine monitoring systems. Researchers have pursued various approaches to obtain the desired cylinder pressure directly or indirectly. However, these approaches vary in cost, reliability, robustness, accuracy and convenience. The use of nonlinear Sliding Observers in pressure and combustion heat release estimation based on measurements of engine speed provides an accurate, low-cost, and reliable way to acquire these desired states.

In this paper the estimation of cylinder pressures and combustion heat releases of a multicylinder SI engine is presented. Since a problem of system observability arises in pressure estimation when the cylinder piston moves to its TDC, means of reducing estimation errors in this condition are described. Finally, the applications of this approach in engine diagnostics are discussed.
\end{abstract}

\section{NOMENCLATURE}

$A_{p} \quad$ piston cross-section area

$e^{\text {piston offset. }}$

$J$ engine moment of inertia about the crankshaft axis.

$L \quad$ length of connecting rod.

$N$ engine speed in rpm.

$P \quad$ cylinder pressure.

$Q$ heat energy.

$R$ crank radius, or half of engine stroke.

$T$ torque or temperature.

$V$ cylinder volume.

$\mathrm{g}$ ratio of specific heats.

(1) angular displacement of engine crankshaft.

w crankshaft velocity.

\section{Subscripts}

$b \quad$ burning.

ch chemical energy in fuel combustion.

d displacement.

Manuscript received November 24, 1993; revised October 20, 1994. Recommended by Guest Editor, G. Rizzoni. This work was supported in part by the Ford Motor Co.

The authors are with Powertrain Control Research Lab, Department of Mechanical Engineering, University of Wisconsin-Madison, Madison, WI 53706 USA.

IEEE Log Number 9408635

\author{
$f$ fuel. \\ fric friction. \\ grav gravity. \\ ht heat transfer. \\ ind indicated. \\ LHV lower heating value. \\ tor torque.
}

\section{INTRODUCTION}

\section{B} ECAUSE of the complexity of today's automotive systems, fault diagnostic tools have become necessary in both on-board electronics and service tools. To satisfy the requirements of the On Board Diagnosis II (OBDII) Regulation, a system is required in passenger cars to monitor components that can cause increased emissions. The appropriate system not only ensures that the engine operates within legislated emission requirements, but also saves time and reduces cost in engine maintenance and failure repair. In addition, some researchers have introduced algorithms for the detection of sensor failures which may give incorrect signals to the engine controller and cause a degradation of engine operation.

Engine misfire detection and identification are important elements in diagnostic systems because engine misfire can induce an increasing level of exhaust gas emission and simultaneously damage the catalytic converter. Many methods have been proposed to solve this problem including algorithms based on variance in engine shaft angular speed, acceleration, or torque, on engine roughness, on spark-plug voltage [24], on the oxygen sensor signal [16], on the knowledge-based expert system, and on neural networks.

Methods using variance in crankshaft velocity, acceleration, torque, or engine roughness have been discussed by Rezeka and Henein [18], Mauer [12], Rizzoni [19], Mihelc and Citron [13], and Klenk et al. [9]. All share the same basic approach, which may be described as follows. There are $n$ firings during one engine cycle for an $n$-cylinder engine. Each firing produces a power pulse to the engine, and these power pulses cause shaft fluctuations in velocity, acceleration, or torque. If we remove the effects of the inertia torque, load torque, and friction and pumping torques, each fluctuation in velocity, acceleration, or torque is related to one wave of combustion power contribution. Theoretically, any abnormality in power contribution will be reflected in the variance in velocity, acceleration, or torque. Thus, examining the variance in velocity or acceleration can provide a measure of cylinder misfire. 
Based on measurement of shaft velocity, these methods offer advantages of simplicity, low equipment cost, and ease of implementation. However, they are usually limited to low speed engine operation. Other methods, such as that using spark plug voltage, have the disadvantage of high hardware cost.

Compared with all these approaches, misfire detection based on the measurement of cylinder pressure is more sensitive and convenient. Examining a cylinder pressure profile permits us to identify cylinder misfire, and is also beneficial for other applications. For example, lean burn engine control often uses cylinder pressure information to calculate the indicated torque [26]. By maintaining the torque fluctuation within an acceptable range, this control system controls the cylinder airfuel ratio within a very limited range. Another example of cylinder pressure measurement applications is feedback for spark timing, fuel, and EGR control [15].

Cylinder pressure can be measured directly or indirectly. Many types of pressure transducers have been developed for the purpose of direct measurement; these provide a very high degree of precision. However, if they are installed in the engines of on-road vehicles to perform control or diagnostic duties, several problems arise. The first is the high cost of the transducer hardware, which prohibits their use in most production on-road vehicles. Although one manufacturer has placed pressure transducers in medium-class commercial vehicles, the installation is only in the first cylinder of the SI engine. A second problem is that the poor durability of current cylinder pressure transducers makes them unfit for production vehicle use. In addition, there is little room in a vehicular engine to install transducers and associated equipment. For these reasons, the alternative of indirect methods to obtain cylinder pressures becomes attractive.

Many studies have measured cylinder pressures indirectly. Citron et al. [2] fed the measured crankshaft velocity into a simple elastic engine model to determine the waveform of indicated torque. This indicated torque was then used to calculate the corresponding cylinder pressure waveform. Rizzoni [20] created an equivalent circuit model to obtain the indicated torque information from the measurement of crankshaft velocity. Connolly and Yagle [3] changed the system independent variable from time to crank angle; with this change, an engine model can be represented by a timevarying linear first-order differential equation instead of a nonlinear differential equation. Using this linear engine model and a stochastic pressure model, the cylinder pressure is reconstructed through a Kalman-filter-based deconvolution algorithm. These methods all use feedforward ways to reconstruct the cylinder pressures. However, large errors often occur because of the noise in actual operation.

Lyon [10] utilized engine vibration signals through a cepstral window to reconstruct the cylinder pressure. Because this method is performed on an off-line basis, however, it is unfit for on-board engine diagnostics. Moreover, some vibration signals due to rough roads or engine accessories reduce the accuracy of this method, and transmissibility of signals (through the engine block, gaskets, etc.) present additional problems.
In contrast to the methods discussed above, pressure estimation by observers has significant advantages. It is a low-cost method, the only equipment needed in the system is a device to measure the engine speed, and minimal engine modification is required. Moreover, the simple structure of Sliding Observers makes them attractive for on-line diagnostic work.

Slotine et al. [25] delineated the theory and structure of nonlinear Sliding Observers, and Misawa and Hedrick [14] provided a useful survey of the observers for nonlinear systems. Although the Extended Kalman Filter is commonly used in state estimation, this filter linearizes the nonlinear system in a piecewise manner, preventing expression of the exact system dynamics and possibly introducing arbitrary large errors. Because the Sliding Observer estimates the system states in a nonlinear system, it may be used to estimate cylinder pressure.

The Sliding Observer is employed for state estimation of many variables, such as vehicle shaft torque [11], intake manifold air pressure [7], cylinder pressure in CI engines [8], and others. In this study such an observer was adapted for estimating the cylinder pressure and combustion heat release of each individual cylinder in a multicylinder SI engine.

\section{Cylinder Pressure and Heat Release Estimation}

Information on the crankshaft speed is very useful for monitoring the internal conditions of an engine. Because of the associated ease of measurement and low hardware cost, crankshaft speed is one of the most frequently used system states for controlling or diagnosing engines, and is employed in the estimation of cylinder pressures presented in this study. This pressure observer has two system states; the instantaneous angular velocity of the crankshaft, which is measurable, and the pressure of the firing cylinder, which is assumed to be nonmeasurable.

To include the most relevant dynamic characteristics of an engine, a nonlinear engine dynamic model with full timevarying engine inertia is employed in the observer. Some engine dynamic models, such as those with constant inertia, cannot provide accurate predictions at high speed engine operation. For this reason, we used a nonlinear engine model in (1) in the observer. The details of this model and its derivation were discussed by Shiao and Moskwa [21] and Shiao et al. [23]

$$
\begin{aligned}
\dot{\omega} & =f_{1}(\theta, \omega, P) \\
& =\frac{1}{J(\theta)}\left(-\frac{1}{2} \frac{d J(\theta)}{d \theta} \omega^{2}+T_{i n d}-T_{\text {fric }}-T_{\text {load }}\right) .
\end{aligned}
$$

In (1), the indicated torque is the total indicated torque for all cylinders. Because a normally firing cylinder has the largest indicated torque at the moment of firing compared with other cylinders, we write the total indicated torque as

$$
\begin{aligned}
T_{i n d} & =\sum_{1}^{n} T_{i n d, i}=\sum_{i}^{n} P_{i} A_{i} L_{t o r, i} \\
& =P_{1} A_{1} L_{t o r, 1}+\sum_{2}^{n} P_{i} A_{i} L_{t o r, i} .
\end{aligned}
$$

$P_{1}$ represents the pressure in the firing cylinder. The effective 


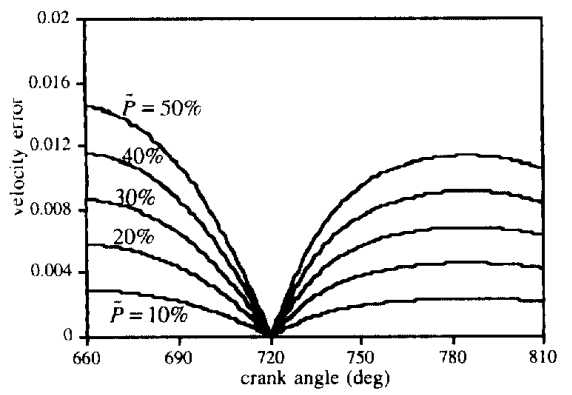

Fig. 1. Velocity errors for different pressure errors at different crank angles. TDC is at $720^{\circ}$.

moment, $L_{t o r}$, for the pressure force about the shaft axis is expressed as

$$
L_{t o r}=R \sin \theta+\frac{R \cos \theta(R \sin \theta+e)}{\sqrt{L^{2}-(R \sin \theta+e)^{2}}} .
$$

An estimated mean friction torque [5] is given in (4), and the load torque can be obtained from the measurement of a torque transducer or estimated by a torque observer

$$
\begin{aligned}
T_{\text {fric }}= & {\left[0.097+0.015\left(\frac{N}{1000}\right)\right.} \\
& \left.+0.005\left(\frac{N}{1000}\right)^{2}\right] V_{d} / 12.56 .
\end{aligned}
$$

The dynamics of cylinder pressure can be simply described by a single zone thermodynamic combustion model. In such a model, it is assumed that the in-cylinder charge is homogeneous, and that the pressure, temperature, and cylinder charge are uniform within the cylinder. Hence, this pressure dynamic equation is written as follows [4], [17]

$$
\dot{P}_{1}=f_{2}\left(\theta, m_{f}, P_{1}\right)=\frac{\gamma-1}{V}\left[\dot{Q}_{c h}-\dot{Q}_{h t}\right]-\frac{\gamma}{V} P_{1} \dot{V} .
$$

The combustion heat release rate, $d Q_{c h}$, can be expressed in terms of the lower heating value and burning rate of the fuel

$$
\frac{d Q_{c h}}{d t}=\dot{Q}_{c h}=\frac{d m_{b}}{d t} Q_{L H V} .
$$

The lower heating value $Q_{L H V}$ can be found in fuel tables. The fuel burning rate is calculated as the product of injected fuel mass and mass fraction burned. Injected fuel mass can be calculated from the fuel flow rate and fuel injection duration, while the mass fraction burned is estimated by a Wiebe function. Therefore, the fuel burning rate is written as

$$
m_{b}=\left(1-\exp \left(-a\left(\frac{\theta-\theta_{0}}{\Delta \theta_{b}}\right)^{m+1}\right)\right) m_{f} .
$$

In (5), the total cylinder volume $V$ consists of the cylinder clearance volume and the swept volume. The value of this volume depends on the piston position as given in (8)

$$
y=L+R-\left(R \cos \theta+\sqrt{L^{2}-(R \sin \theta+e)^{2}}\right) .
$$

Thus, this cylinder volume and its rate are written as

$$
\begin{aligned}
& V=V_{\text {clear }}+y A_{p}, \\
& \dot{V}=\dot{y} A_{p}=A_{p} L_{\text {tor }} \omega .
\end{aligned}
$$

Equations (1) and (5) describe the dynamics of the true system states. We use the symbols $\hat{\omega}$ and $\hat{P}_{1}$ to represent the estimates of the two system states $\omega$ and $P_{1}$. Based on the speed and pressure dynamic models in (1) and (5), as well as modification of the switching term, the structure of a Sliding Observer for pressure estimation is defined as

$$
\begin{aligned}
\dot{\hat{\omega}} & =\hat{f}_{1}-\alpha_{1} \tilde{\omega}-K_{\omega} \operatorname{sat}(\tilde{\omega} / \eta), \\
\dot{\hat{P}}_{1} & =\hat{f}_{2}-\alpha_{2} \tilde{\omega}-K_{p} \operatorname{sat}(\tilde{\omega} / \eta)
\end{aligned}
$$

where sat is a saturation function defined as [14]

$$
\operatorname{sat}(\tilde{\omega} / \eta)=\left\{\begin{array}{ll}
\tilde{\omega} /|\tilde{\omega}| & \text { if }|\tilde{\omega}| \geq \eta \\
\tilde{\omega} / \eta & \text { if }|\tilde{\omega}|<\eta
\end{array} .\right.
$$

Using this saturation function instead of the signum function used by Masmoudi and Hedrick [11] and Kao and Moskwa [8] allows us to avoid chattering errors across the sliding surface and provides smooth dynamics and control.

If $\Delta f$ represents the modeling error between the observer model $\hat{f}$ and the true model $f$, the dynamics of estimation errors are given by (14) and (15)

$$
\begin{aligned}
\dot{\tilde{\omega}} & =\Delta f_{1}-\alpha_{1} \tilde{\omega}-K_{\omega} \operatorname{sat}(\tilde{\omega}), \\
\dot{\tilde{P}}_{1} & =\Delta f_{2}-\alpha_{2} \tilde{\omega}-K_{p} \operatorname{sat}(\tilde{\omega}) .
\end{aligned}
$$

The function of the linear term gain $\alpha_{i}$ is to help the estimate reach the sliding patch (similar to the Extended Kalman Filter), while the switching gains $K_{p}$ and $K_{\mathrm{w}}$ maintain the system dynamics in sliding, and make the boundary layer attractive. The determination of these gains has been discussed by Kao and Moskwa [8] and Shiao and Moskwa [22].

The sliding surface in the Sliding Observer is defined as the difference $(\tilde{\omega})$ between the measured and estimated engine speeds. During sliding, because the condition of $s_{1}=\tilde{\omega}=0$ is satisfied, the system dynamics are reduced from those of a second order system to those of a first order system

$$
\hat{\tilde{P}}_{1}=-\frac{K_{p}}{K_{\omega}} \Delta f_{1}+\Delta f_{2}
$$

Therefore, keeping the dynamic errors on the sliding surface will result in good estimation of cylinder pressure if the gains $K_{p}$ and $K_{\mathrm{w}}$ are well designed.

Meanwhile, we can estimate the corresponding combustion heat release for the firing cylinder. The heat release rate is given by a single zone combustion model as

$$
d \hat{Q}_{c h}=\frac{\gamma}{\gamma-1} \hat{P} d V+\frac{1}{\gamma-1} V d \hat{P}+d Q_{h t} .
$$

Subsequently, the combustion heat release can be computed by integrating the release rate in (17)

$$
\hat{Q}_{c h}=\int_{t_{0}}^{t} \dot{\hat{Q}}_{c h} d t
$$



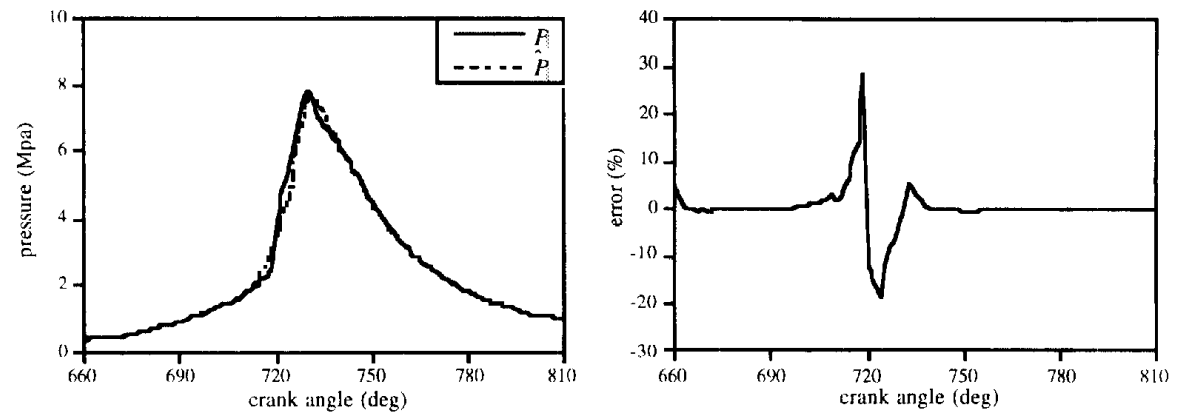

Fig. 2. Estimated cylinder pressure, measured pressure and estimation error for a high pressure condition with parameters $a=5 . m=2$. and $\theta_{0}=705^{\circ}$.
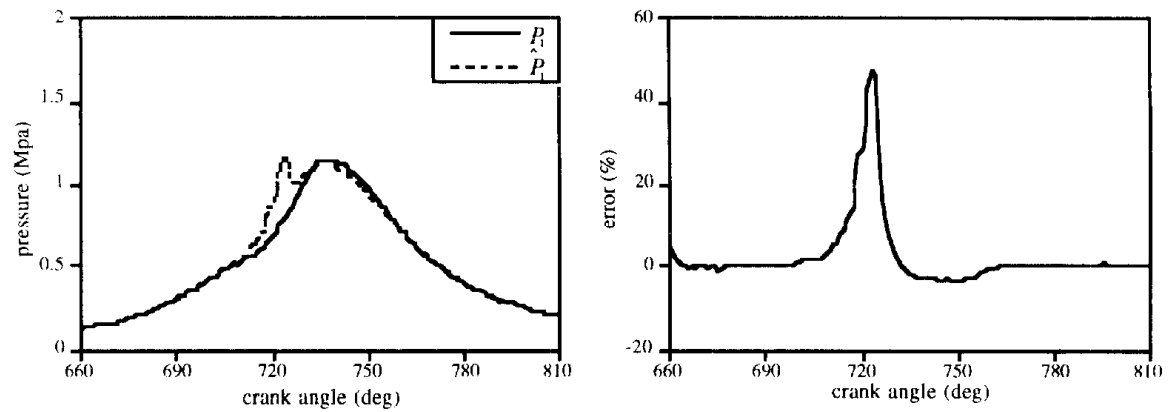

Fig. 3. Estimated cylinder pressure, measured pressure and estimation error for a low pressure condition with parameters $a=5, m=2$, and $\theta_{0}=705^{\circ}$.
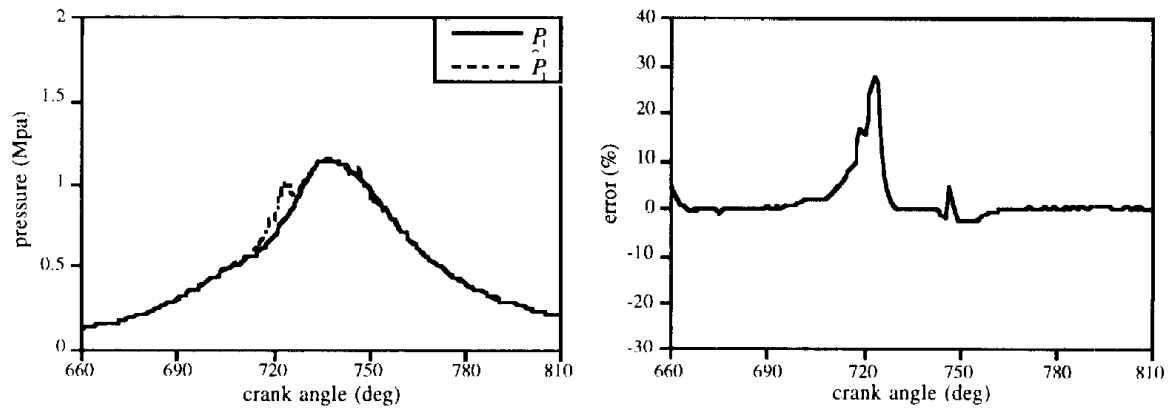

Fig. 4. Estimated cylinder pressure, measured pressure and estimation error for a low pressure condition with parameters $a=3, m=2$, and $\theta_{0}=70.3^{\circ}$

\section{SYSTEM OBSERVABILITY}

Pressure estimation of this Sliding Observer is accomplished by using the error between the measured and estimated crankshaft velocities as feedback to reduce the dynamic error in estimated pressure. To assure good estimation of cylinder pressure, the system observability must be always maintained so that this feedback is performed successfully. From the equation of engine dynamics, (1), we see that the observability strongly depends on the effective moment arm $L_{t o r}$ of the indicated torque. If this arm becomes zero, there will be no feedback path from which the velocity errors can correct the pressure estimation. In other words, for this two-state system to be observable, the $f_{1}$ function (1) has to be a single valued function of the state $P_{1}$. This means $\Delta f_{1}$ in (16) must be a function of $P_{1}$ so that the first term $-\left(K_{p} / K_{\omega}\right) \Delta f_{1}$ can influence the error dynamics [25]. Unfortunately, there is no moment arm when the cylinder piston moves to its TDC or BDC positions. This is apparent from (3) if the shaft angle $\theta$ is zero or $180^{\circ}$ and the piston has no offset. Under this condition, $f_{1}$ is no longer a function of $\Gamma_{1}$. Thus the change in $\Delta f_{1}$ has no influence on the error of $P_{1}$, meaning that the cylinder pressure can be estimated only by (5) at TDC position, and there is no feedback from (6). The modeling error in (5) completely determines the magnitude of the pressure error.

An explanation of this unobservability of TDC is provided in Fig. 1. For the region around TDC position $\left(720^{\circ}\right)$, pressure errors and their resulting velocity errors are plotted for each crank angle. At TDC, the velocity errors are always equal to zero even though the pressure errors increase to high values. Under this condition, the observer system loses its observability and it becomes impossible to use velocity error 

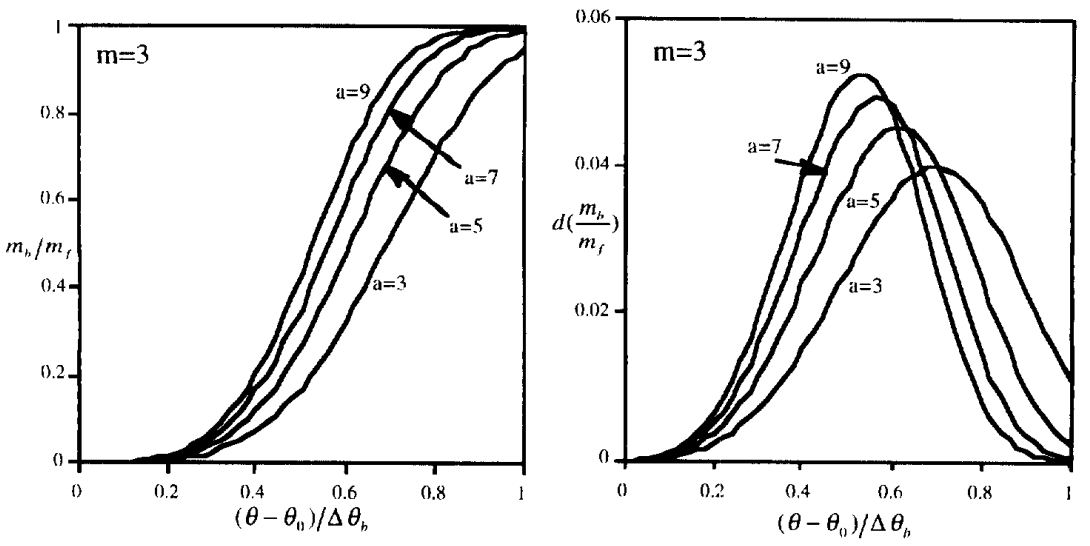

Fig. 5. Wiebe function for mass fraction burned and its rate with varying efficiency parameter $a$.
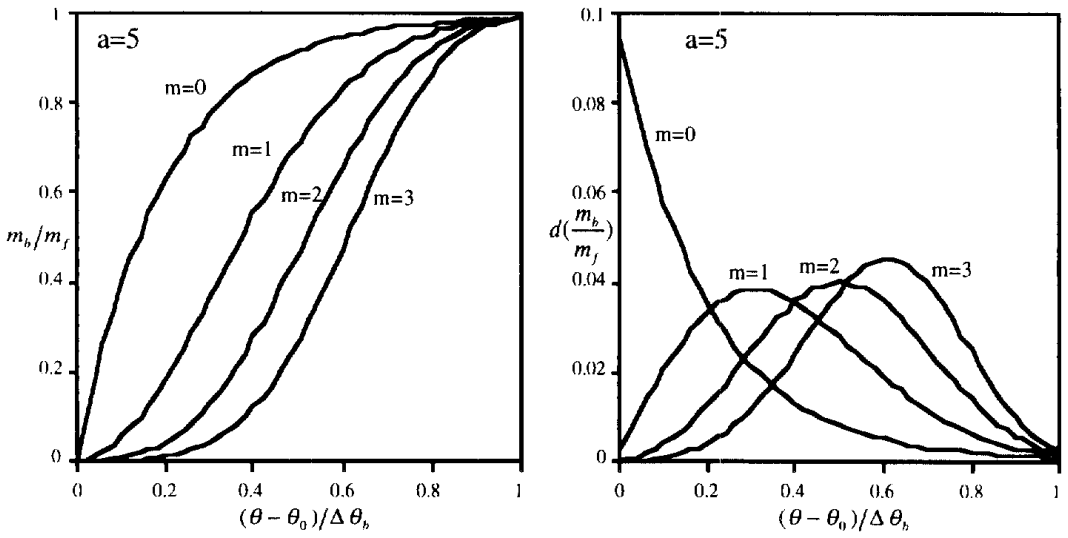

Fig. 6. Wiebe function for mass fraction burned and its rate with varying form factor $m$.
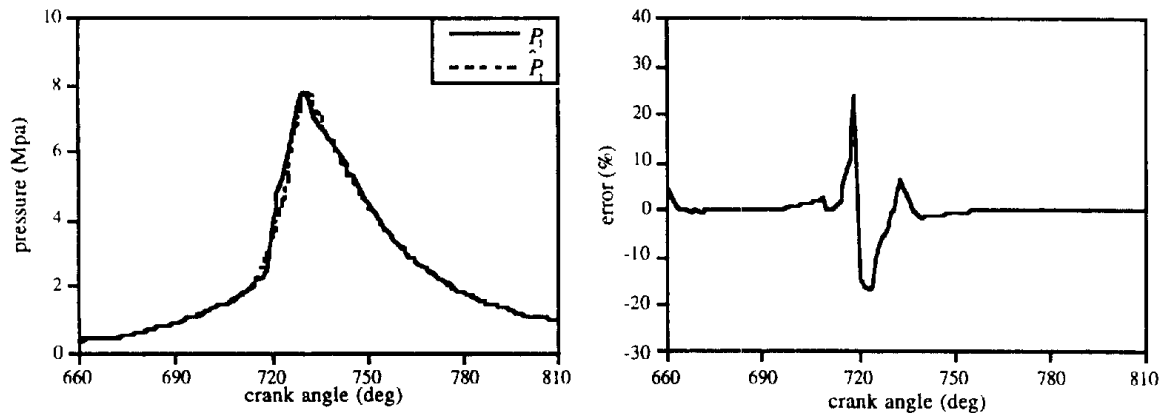

Fig. 7. Estimated cylinder pressure, measured pressure and estimation error for a high pressure condition with parameters $a=10, m=3$. and $\theta_{0}=705^{\circ}$

as feedback to correct the pressure estimation error at this position. At crank angles near TDC, the errors in velocity are not very significant, although the pressure errors are large. This explains why large pressure errors can occur around the TDC position even though the estimated velocity is very close to the measured velocity.

\section{OBSERVER PARAmeters}

One way to solve the problem of large pressure errors around TDC is to reduce the modeling errors in the pressure dynamic equation (5). In the combustion model, the dynamics of cylinder pressure are determined by the combustion heat release rate, cylinder volume change and cylinder pressure. Because the cylinder volume change rate is determined by the engine configuration and speed, the only factor that influences modeling accuracy is the given heat release rate model (the Wiebe function).

According to (7), the characteristics of the heat release rate depend on the injected fuel mass $m_{f}$, the start angle of combustion $\theta_{0}$, the total combustion duration $\Delta \theta_{b}$, the 


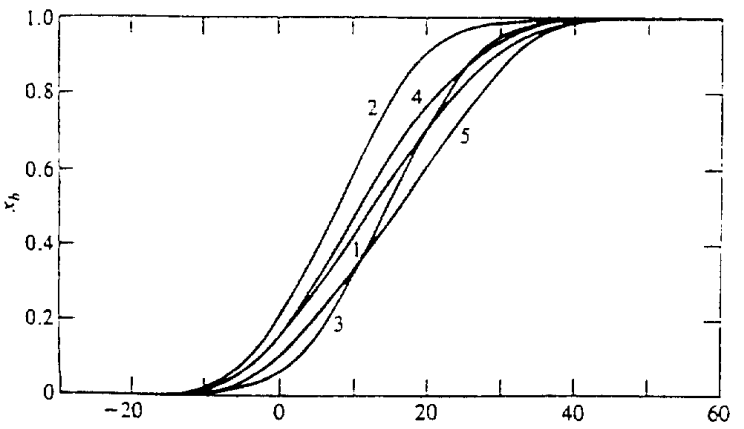

Fig. 8. Cylinder mass fraction burned for five consecutive cycles.

efficiency parameter $a$, and the form factor $m$. The injected fuel mass is determined by the engine control module. It is also reasonable to maintain the combustion duration $\Delta \theta_{b}$ as constant. Therefore, the start angle of combustion, the efficiency parameter and the form factor largely control the accuracy of combustion models.

Heywood et al. [6] used the Wiebe function in (7) to calculate the mass fraction burned of the charge within the cylinder for each crank angle. These authors examined several values of $a$ and $m$ to obtain a good match for the experimental pressure curves, and concluded that the set of $a=5$ and $m=2$ can provide a reasonable estimate for many conditions. The values suggested in their study can indeed achieve a reasonably good match for pressure curves at full range operation, but they are not optimal values at specific low or high pressure conditions. To test the values of $a$ and $m$. we used measured cylinder pressures and the engine configuration of a Ford $4.6 \mathrm{~L} \mathrm{~V}-8$ engine to examine the designed pressure observer. For high cylinder pressure, the estimation using the Sliding Observer with parameters $a=5$ and $m=2$ is shown in Fig. 2. The suggested parameters $a$ and $m$ match the measured cylinder pressure, so that the pressure errors at the crankshaft angles around TDC are not large. However, large estimation errors occur if the same parameters $a$ and $m$ are applied with low cylinder pressure. These errors, shown in Fig. 3, indicate that these parameters are unfit for very low pressure conditions. In contrast, another set of parameters, $a=3$ and $m=2$ (Fig. 4), significantly reduces the estimation errors at these angles. These examples indicate that observer design must utilize adjustable $a$ and $m$, whose values depend on the pressure levels. Several fraction burned curves and the rate of fraction change are plotted in Figs. 5 and 6 for various $a$ and $m$ [6]. These figures show the effect of varying $a$ and $m$. For a fixed form factor $m$ (Fig. 5), the larger the value of $a$, the more rapid the fraction change occurring around the midpoint of the total combustion duration. The maximum heat release rate shifts to the first half of the combustion period. For a fixed efficiency parameter $a$ (Fig. 6), a larger $m$ value causes the second half of the combustion period to have a larger heat release. According to the effects of these two parameters and the analysis of experimental data, a high efficiency parameter and form factor are suggested for high pressure cycle operation, while estimation in a low pressure cycle can be modeled with a low parameter $a$ and low factor $m$. This cycle-tocycle variation in parameters can cause the estimation errors in (7) to become small. Pressure estimation using the Sliding Observer with different parameters at high and low pressure cycles is shown in Figs. 7 and 4, respectively. High values $a=10$ and $m=3$ are employed in Fig. 7. and low values $a=3$ and $m=2$ in Fig. 4. Compared with previous pressure estimation results, this parameter modification offers improved performance.

Of course, because of combustion variation, the shapes of fraction burned or heat release rates are different from cycle to cycle. The mass fraction burned for five consecutive cycles in an SI engine is shown in Fig. 8 [1]. The shape of each curve is accompanied by a set of $a$ and $m$. Even though $a$ and $m$ are adjusted for different pressure levels, combustion variations can still cause modeling error to produce errors in pressure estimation. We may use the information about the estimated combustion heat release and its rate at the early combustion stage to identify an appropriate $a$ and $m$, and apply these values in the observer for the remainder of combustion. However, the early stage of combustion is usually close to the TDC position. Thus, not many data are available to identify the $a$ and $m$ in the current heat release curve. Nevertheless, the adjustable $a$ and $m$ can reduce some estimation errors for the weak or nonobservable periods.

An alternative means to reduce the modeling error is to adjust the combustion start angle $\theta_{0}$. This angle is different for low and high speed operations. In Fig. 9 a pressure estimation similar to that in Fig. 4 is shown, but with a different combustion start angle. This new start angle causes the errors in the estimation to be smaller than those in Fig. 4. After the cylinder pressure and combustion heat release are estimated, an actual combustion start angle can be observed. Replacing the originally designed start angle, this observed angle can reduce pressure estimation error at TDC for the next combustion cycle. Sometimes a cylinder may exhibit abnormal combustion (i.e., late burning) in one cycle but may return to normal in the next cycle. If the combustion start angle is changed after the abnorrnal combustion, then the new start angle is applied in the next cycle. Because this second cycle has a normal combustion, the changed start angle does not improve pressure estimation error at TDC; instead, it degrades the estimation. Therefore, the start angle is changed only after two or three consecutive abnormal combustion cycles are observed. Otherwise, the original start angle is still used.

\section{Misfire and Combustion Fault Detection}

The previously described pressure observer can be used as a detection tool for misfire or other abnormal combustion. Ideally, the cylinder pressures estimated by the observer are close to the measured pressures under any combustion conditions. If this was true, it would be easy to recognize cylinder misfire or abnormal combustion by setting some varying thresholds around the pressure profiles. However, actual conditions are somewhat different. Because of the nonobservability problem 

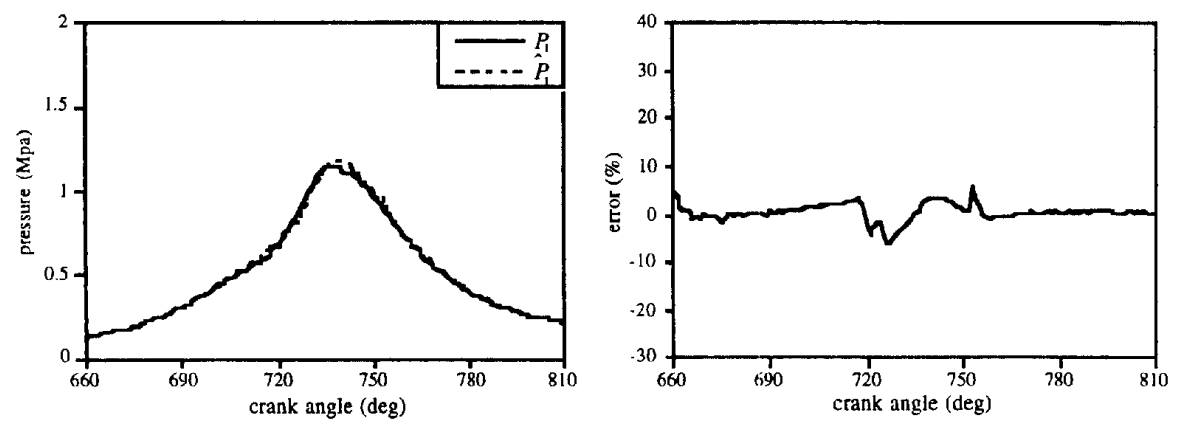

Fig. 9. Estimated cylinder pressure, Measured pressure and estimation error for a low pressure condition with parameters $a=3, m=2$, and $\theta_{0}=712^{\circ}$.

occurring around TDC, the estimated pressure is not close to the measured pressure profile if there is a full or partial misfire. It is difficult to use an estimated misfire pressure with large error for misfire detection by setting a threshold around the pressure profile. If we assume that there is a permanent cylinder misfire in a cylinder, and we can detect the first misfire cycle right after it occurs, then we can change the parameters or the injected fuel mass in (7). Thus, in the next combustion cycle, the estimation of pressure dynamics will be closer to the measured pressure under misfire. One question arises in applying this method: How can we detect the misfire near the time when it begins? If the cylinder is just in abrupt misfire and return to a normal combustion condition, changing the model parameters will cause the estimation for the following normal cycle to produce a large estimation error.

Now let us suppose that a misfire cycle occurs right after a normal combustion cycle, and that the parameters used in the previous normal cycle are still used in this misfire cycle. The measured and estimated cylinder pressures in a misfired cylinder are shown in Fig. 10. For the angles far from the TDC position, because of the strong observability, good pressure estimation is obtained even though the parameters in (7) are for a normal combustion condition. However, when the piston goes toward or just passes its TDC position, weak or zero observability causes large estimation errors. For the reasons mentioned previously, there is no feedback from the errors between measured and estimated velocities to the pressure estimation at TDC. The prediction of cylinder pressure is determined entirely by the pressure equation in (5). Hence, the magnitude of the modeling error in (5) determines the magnitude of the pressure estimation error. In this abrupt misfire cycle, the parameters of the pressure model in (5) are designed for normal combustion, not for a misfiring cylinder. Undoubtedly, the large modeling error will cause the observer to produce large pressure estimation errors at angles with weak or no observability. Therefore, the estimated cylinder pressure profile deviates significantly from the measured pressure. No proper thresholds can be set around the profile of this incorrectly estimated pressure to detect misfire.

Utilizing the combustion heat release and release rate estimation, a solution to this problem is achieved. The heat release and release rate for the estimated misfire pressure are given in Fig. 11. For a typical case of combustion under misfire, since

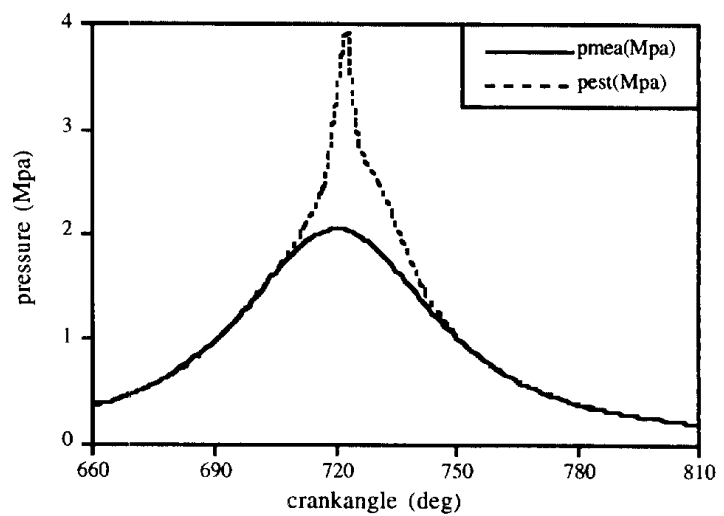

Fig. 10. Estimated cylinder pressure and measured pressure of a misfired cylinder with parameters $a=5, m=2$, and $\theta_{0}=705^{\circ}$.

there is no heat discharged from the mixture, the heat release is near zero for every crank angle. The corresponding heat release rate also has zero value for each angle. The estimated heat release in Fig. 11 is quite different from the typical profile. In Fig. 11, the estimated heat release has zero values at the beginning. After the angle passes the combustion start angle and moves closer to TDC, the estimated heat release rate begins to increase above zero. This apparent increasing heat release rate causes the observer to have a higher cylinder pressure than the measured pressure. Subsequently, higher estimated crankshaft velocity and high velocity error result from this higher estimated pressure. The observer can force the estimated velocity to return to the sliding surface of zero velocity error, but weak observability prevents the observer from correcting the pressure errors. Since the heat release and release rate continue to increase to their maximum values under the parameters for normal combustion, the estimated pressure continues to deviate from the measured pressure. Consequently, large pressure errors are produced.

After the TDC position is passed, observability returns and the feedback relationship between pressure error and velocity error gradually becomes stronger. Thus, the pressure error is once again under control, and the estimated pressure becomes closer to the measured pressure. The estimated heat release and rate also decrease and approach the actual values, which 


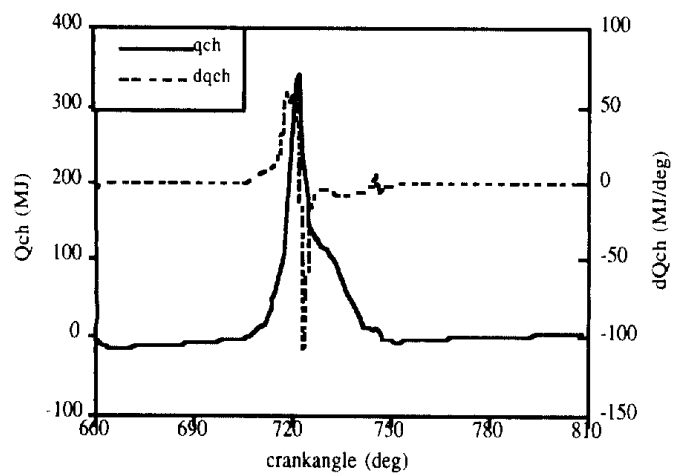

Fig. 11. Estimation of combustion heat release and release rate of a misfired cylinder.

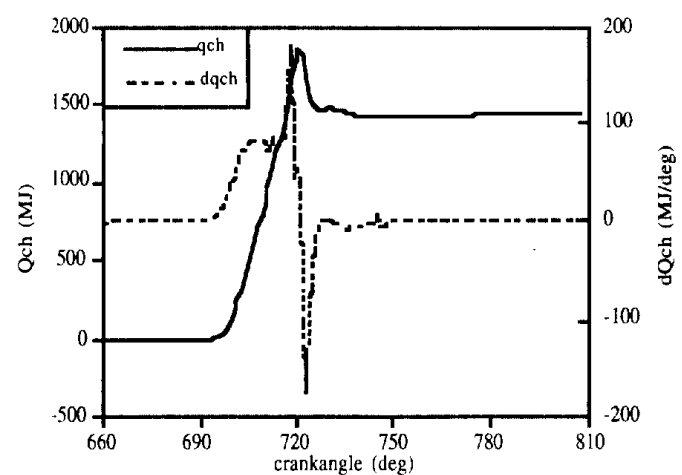

Fig. 12. Estimation of combustion heat release and release rate of pre-ignition combustion.

are zeros for misfire. As a result, the estimated heat release increases above zero value before TDC and drops back to zero, while zero is the value for actual total heat release in a misfiring cylinder. This characteristic of increasing and decreasing heat release reflected in its release rate is a positive jump to discharge energy and a negative peak to reabsorb the energy. Based on this characteristic, detection of misfire becomes easy. If the estimated heat release is observed to have a zero final value at an angle far from TDC, this cylinder is concluded to be misfiring. Alternatively, if a peak in the release rate curve with a negative value follows a positive peak value, a misfiring cylinder is identified. The heat release and its rate in a partial misfire case will have similar contours to those in a full misfire case, but with a nonzero final value of heat release at the end of combustion. Therefore, the heat release contour can be used to identify a partial misfire. From the ratio of the final heat release value of partial misfire to the nominal value, the percentage of misfire can be approximately calculated.

Similarly, this method can be applied in the detection of abnormal combustion, such as pre-ignition or late burning. Because of unobservability, the heat release rates in preignition (Fig. 12) or late burning (Fig. 13) have unique release patterns. Also, the estimated total heat release is less than the nominal heat release if a partial misfire occurs. The

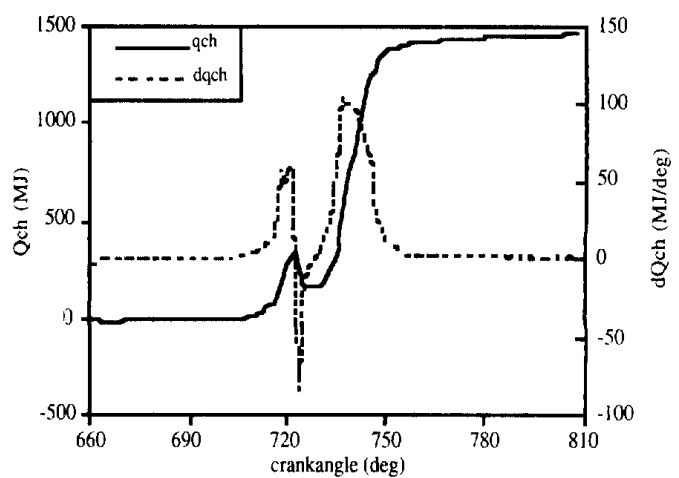

Fig. 13. Estimation of combustion heat release and release rate of late-burning combustion.

characteristics of these patterns are used as indications of abnormal combustion.

\section{CONClusion}

The study introduced the use of a Sliding Observer for estimation of cylinder pressure and combustion heat release for SI engines. In this estimation, the unobservability problem at piston TDC position may induce large apparent pressure estimation errors. However, adjustable parameters in the observer are used to improve the accuracy. Moreover, the detection of cylinder misfire or abnormal combustion is also achieved by utilizing the estimated heat release or heat release rate. A load torque observer can be added to the engine speed dynamic equation to further reduce modeling errors.

\section{ACKNOWLEDGMENT}

The authors wish to acknowledge Drs. W. J. Johnson, D. Hrovat, and K. Marko of the Ford Scientific Research Laboratory for providing technical support and hardware. Also, Drs. G. Lawson, K. Dobbins, and Mr. J. Dosdall contributed engine data, hardware, and time to this effort.

\section{REFERENCES}

[1] G. P. Beretta, M. Rashidi, and J. C. Keck, "Turbulent flame propagation and combustion in spark ignition engines," Combustion Flame, vol. 52. pp. 217-245, 1983.

(2) S. J. Citron, J. E. O'Higgins, and L. Y. Chen, "Cylinder by cylinder engine pressure and pressure torque waveform determination utilizing speed fluctuations," SAE Tech. paper 890486, 1989.

[3] F. T. Connolly and A. E. Yagle, "Modeling and identification of the combustion pressure process in internal combustion engines using engine speed fluctuations," ASME DSC-vol. 44, 1992.

[4] J. A. Gatowski, E. N. Balles, K. M. Chun, F. E. Nelson, J. A. Ekchian, and J. B. Heywood, "Heat release analysis of engine pressure data," SAE Tech. paper 841359, 1984.

[5] J. B. Heywood, Internal Combustion Engine Fundamentals. New York: McGraw-Hill, 1989.

[6] J. B. Heywood, J. M. Higgins, P. A. Watts, and R. J. Tabaczynski. "Development and use of a cycle simulation to predict SI engine efficiency and $\mathrm{NO}_{\mathrm{x}}$ emissions." SAE Tech. paper 790291. 1979.

[7] P. Kaidantzis, P. Rasmussen, M. Jensen, T. Vesterholm, and E. Hendricks." "Advanced nonlinear observer control of SI engines," SAE Tech. paper 930768, 1993.

[8] M. Kao and J. J. Moskwa, "Nonlinear turbocharged diesel engine control and state observation," ASME Winter Annual Meeting. New Orleans, LA., DSC-vol. 52, 1993, pp. 187-198. 
[9] M. Klenk, W. Moser, W. Mueller, and W. Wimmer, "Misfire detection by evaluating crankshaft speed-A means to comply with OBDII," SAE Tech. paper $930399,1993$.

[10] R. H. Lyon, Machinery Noise and Diagnostics. Butterworth Publishers, 1987.

[111 R. A. Masmoudi and J. K. Hedrick, "Estimation of vehicle shaft torque using nonlinear observers," ASME Winter Annual Meeting, Boston, MA, Dec. 13-18, 1987

[12] G. F. Mauer, "On-line cylinder fault diagnostics for internal combustion engines," IEEE Trans. Indust. Electron., vol. 37, no. 3, pp. 221-226, 1990.

[13] W. P. Mihelc and S. J. Citron, "An on-line engine roughness measurement technique," SAE Tech. paper 840136, 1984.

[14] E. A. Misawa and J. K. Hedrick, "Nonlinear observers-A state-of-theart survey," ASME J. Dynamic Systems, Measurement, and Control, vol. $111,1989$.

[15] G. W. Pestana, "Engine control methods using combustion pressure feedback," SAE Tech. paper 890758, 1989.

[16] G. Plapp, M. Klenk, and W. Moser, "Methods of on-board misfire detection," SAE Tech. paper 900232, 1990.

[17] J. I. Ramos, Internal Combustion Engine Modeling. Hemisphere, 1989.

[18] S. F. Rezeka and N. A. Henein, "A diagnostic technique for the identification of misfiring cylinder(s)," SAE Tech. paper 870546, 1987.

[19] G. Rizzoni, "Diagnosis of individual cylinder misfires by signature analysis of crankshaft speed fluctuations," SAE Tech. paper 890884 , 1989.

[20] _Estimate of indicated torque from crankshaft speed fluctuations: A model for the dynamics of the IC engine," IEEE Trans. Veh. Technol., vol. 38 , no. 3 , pp. $168-179,1989$.

[21] Y. Shiao and J. J. Moskwa, "An investigation of load force and dynamic error magnitude using the lumped mass connecting rod model," SAE Tech. paper 930617,1993

[22] ___ "Misfire detection and cylinder pressure reconstruction for SI engines," SAE Tech. paper 940144, 1994

[23] Y. Shiao, C. H. Pan, and J. J. Moskwa, "Advanced dynamic S.I. engine modeling for diagnostics and control," Int.J. Vehicle Design, vol. 15 no. $6,1994$.

[24] Y. Shimasaki, M. Kanehiro, S. Baba, S. Maruyama, T. Hisaki, and S. Miyata, "Spark plug voltage analysis for monitoring combustion in an internal combustion engine," SAE Tech. paper 930461, 1993.
25] J. J. E. Slotine, J. K. Hedrick, and E. A. Misawa, "On sliding observers for nonlinear systems," ASME J. Dynamic Systems, Measurement, and Control, vol. 109, 1987.

[26] N. Sugitani, M. Ueda, and M. Kosugi, "Combustion pressure sensor for Toyota lean burn engine control," SAE Tech. paper 930882, 1993.

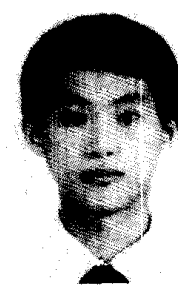

Yaojung Shiao received the B.S. degree in 1986 from the Department of Mechanical Engineering, National Cheng-Kung University, Tajwan, R.O.C., and the M.S. degree in 1990 from the Department of Mechanical Engineering, University of Wisconsin-Madison.

$\mathrm{He}$ is a Ph.D. candidate in the Department of Mechanical Engineering, University of Wisconsin-Madison.

He is a member of SAE and ASME.

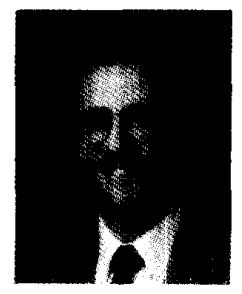

John J. Moskwa received the B.S.E. and M.S.E. degrees from the University of Michigan in 1980 and 1981 , respectively, and the Ph.D. degree from MIT in 1988.

Dr. Moskwa is Assistant Professor and Director of the Powertrain Control Research Laboratory at the University of Wisconsin-Madison. Prior to coming to MIT he was Senior Engineer in the Experimental Mechanics Laboratory at Cummins Engine Company (1981-1984), and has also worked at GM Research Laboratories $(1985,1986)$ and at the US Anny Tank Automotive Command Research Engineering and Development Center (TARDEC, 1991). His current research interests are in the areas of vehicular powertrain system modeling, diagnostics and controls, and he has been involved professionally in the powertrain area for over 20 years. 